\title{
Toll-like receptor 3 signaling induces apoptosis in human head and neck cancer via survivin associated pathway
}

\author{
NOZOMI NOMI, SATORU KODAMA and MASASHI SUZUKI \\ Department of Otolaryngology, Oita University Faculty of Medicine, 1-1 Idaigaoka, \\ Hasama-cho, Yufu, Oita 879-5593, Japan
}

Received December 29, 2009; Accepted March 2, 2010

DOI: 10.3892/or_00000850

\begin{abstract}
The innate immune system builds up host defense against a huge diversity of pathogens, such as viruses, bacteria and fungi. Toll-like receptors (TLRs) are one of the pattern recognition receptors that trigger the initiation of various defense mechanisms. The expression of TLRs represents an important link between innate and adaptive immune responses. The expression of TLRs in several cancer cell types has been reported; however, the exact roles they play in cancers are still unclear. In this study, we investigated the expression and signaling of TLRs in human head and neck squamous cell carcinomas (HNSCCs). The expression of TLRs in 8 HNSCC cell lines was examined by Western blot analysis and flow cytometry. The expression of TLR mRNA was also examined by quantitative RT-PCR. Cells were incubated with poly I:C, a TLR3 ligand, and cell viability was tested by MTT assay. In addition, apoptosis was analyzed by flow cytometry with Annexin V and propidium iodide staining. The expression of survivin, a member of the inhibitors of the apoptosis protein family, was also investigated. TLR2 and TLR3 were widely expressed in human HNSCCs. Interestingly, the stimulation of TLR3 by poly I:C induced apoptosis in cancer cells in a dose-dependent manner. The expression of survivin was down-regulated during apoptosis, suggesting that TLR signaling affects survivinmediated signal transduction in apoptosis. In the present study, we demonstrated the proapoptotic activity of TLR3 expressed by HNSCCs. These results suggest that TLR3 could be a new target for therapy in HNSCCs.
\end{abstract}

\section{Introduction}

The innate immune system builds up host defense against a huge diversity of pathogens, such as viruses, bacteria and

Correspondence to: Dr Satoru Kodama, Department of Otolaryngology, Oita University Faculty of Medicine, 1-1 Idaigaoka, Hasama-cho, Yufu, Oita 879-5593, Japan

E-mail: satoruk@med.oita-u.ac.jp

Key words: innate immunity, toll-like receptor 3, head and neck cancer, apoptosis, survivin fungi. Toll-like receptors (TLRs) are one of the pattern recognition receptors that trigger the initiation of various defense mechanisms. Ligation of TLRs by their respective ligands triggers well-characterized signaling cascades that result in activation of downstream effectors, resistance against pathogens and, occasionally, cell death (1-3). In humans, 11 different TLRs, which are expressed on various immune cells and a variety of stromal cells, have been described. The expression of TLRs represents an important link between innate and adaptive immune responses. Among the TLRs, TLR3, TLR7, TLR8 and TLR9 recognize nucleic acids; TLR7 and TLR8 recognize single stranded RNA (ssRNA) $(4,5)$ or shortinterfering RNA (siRNA) (6); TLR9 recognizes DNA containing unmethylated $\mathrm{CpG}$ motifs (7); and TLR3 recognizes dsRNA derived from viruses or host RNA $(8,9)$. TLR3 is known not to participate in the MyD88 signaling pathway, but in the Toll/IL-1R domain-containing adapter-inducing IFN-B (TRIF)-dependent pathway. TLRs act in protective innate immunity, and their expression is observed in immunocompetent and epithelial cells.

The expression of TLRs in several cancer cell types has been reported; however, their exact roles in cancers are still unclear. Of the TLRs, TLR3-signaling directly induces apoptosis in a variety of cancer cell lines, including breast cancer, hepatocellular cancer, cervical cancer, colon cancer and melanoma (10-14). The expression of TLRs and the association of gene polymorphisms of TLR in HNSCC have also been reported $(15,16)$; however, TLR signaling by ligand stimulation in HNSCCs has not yet been closely investigated. In this study, we investigated the expression and signaling of TLRs in HNSCC cell lines, particularly TLR3, and extensively analyzed the proapoptotic activity of TLR3.

\section{Materials and methods}

Cell lines. Human tumor cell lines HEp2 (laryngeal squamous cell carcinoma) and SCC25 (tongue squamous cell carcinoma) were obtained from Dainippon Sumitomo Pharmacology (Tokyo, Japan). The human tumor cell lines HSC2 (oral squamous cell carcinoma), HSC3 (tongue squamous cell carcinoma), HSC4 (tongue squamous cell carcinoma), SAS (tongue squamous cell carcinoma), HSQ89 (maxillary sinus squamous cell carcinoma), and HO-1-u-1 (oral squamous cell carcinoma) were obtained from Riken BRC Cell Bank (Ibaragi, Japan). 
Cell cultures. HEp2, HSC2 and HSC3 were cultured in $3 \mathrm{ml}$ of MEM- $\alpha$ (Gibco, NY, USA) plus 10\% (v/v) heat-inactivated fetal bovine serum (FBS, Gibco) in $25 \mathrm{~cm}^{2}$ plates at $1 \times 10^{6}$ cells/plates. HSC4, SAS and HO- $1-\mathrm{u}-1$ were also cultured in $3 \mathrm{ml}$ of RPMI-1640 (Gibco) plus 10\% FBS. SCC25 cells were cultured in $3 \mathrm{ml}$ of DMEM/F12 (diluted 1:1, Gibco) plus $10 \%$ FBS. DMEM/F12 was supplemented with L-glutamine $(15.82 \mathrm{ml} / \mathrm{l})$. HSQ89 cells were cultured in $3 \mathrm{ml}$ of DMEM. Cells were cultured at $37^{\circ} \mathrm{C}$ in a humidified incubator containing $5 \% \mathrm{CO}_{2}$.

RNA extraction and reverse transcription. Total RNA was isolated from cell lines using the RNeasy Mini kit (Qiagen, Hilden, Germany), following the manufacturer's instructions. Cells were washed twice with PBS and lysed with $1 \mathrm{ml}$ $0.25 \%$ trypsin/EDTA. All cells were used at $70 \%$ confluence. The quantity and quality of the extracted RNA was determined by spectrophotometry.

Reverse transcription was conducted using TaqMan Reverse Transcription Reagents (PE Applied Biosystems, Foster City, CA, USA), according to the manufacturer's protocol. Briefly, $1 \mu \mathrm{g}$ of the total RNA was dissolved in $38.5 \mu \mathrm{l}$ of DEPC-treated $\mathrm{H}_{2} \mathrm{O}$ as the template. Further constituents per $100 \mu \mathrm{l}$ were as follows: $10 \mu \mathrm{l}$ of $10 \mathrm{X}$ TaqMan RT buffer, $22 \mu \mathrm{l}$ of $25 \mathrm{mM} \mathrm{MgCl}_{2}, 20 \mu \mathrm{l}$ of $2.5 \mathrm{mM} \mathrm{dNTP}$ mixture, $5 \mu 1$ of $50 \mathrm{mM}$ random hexamer, $2 \mu 1$ of $20 \mathrm{U} / \mathrm{ml}$ RNase inhibitor, $2.5 \mu 1$ of $50 \mathrm{U} / \mathrm{ml} \mathrm{MultiScribe}$ reverse transcriptase. This mix was subsequently incubated at $25^{\circ} \mathrm{C}$ for $10 \mathrm{~min}, 48^{\circ} \mathrm{C}$ for $30 \mathrm{~min}$ and $95^{\circ} \mathrm{C}$ for $5 \mathrm{~min}$, and stored at $4^{\circ} \mathrm{C}$. Synthesized cDNA $(2 \mu \mathrm{l})$ was used for each PCR reaction.

RT-PCR analysis. Primer sequences were as follows; GAPDH-forward (F), 5'-GCATCCTCACCCTGAAC-3'; GAPDH-reverse (R), 5'-TTCTCCTTAATGTCACCCAC-3'; TLR2-F, 5'-GGCTTCTCTGTCTTGTGACC-3'; TLR2-R, 5'GGGCTTGAACCAGGAAGACG-3'; TLR3-F, 5'-AGCC ACCTGAAGTTGACTCAGG-3'; TLR3-R, 5'-CAGTCAA ATTCGTGCAGAAGGC-3'; TLR4-F, 5'-CGCCTTGCGA GCAGGAACACTTAC-3'; TLR4-R, 5'-GAACAGCGCCA CCTGCTGCCTGAG-3'; TLR9-F, 5'-TACCAACATCCTG ATGCTAGACTC-3'; TLR9-R, 5'-TAGGACAACAGCAG ATACTCCAGG-3'; survivin-F, 5'-ATGGCCGAGGCTGG CTTCATCCAC-3'; survivin-R, 5'-TTGTAGTTTGTGCTA TTCTGTGAATTA-3'; GAPDH (control for survivin)-F, 5'ATTCCATGGCACCGTCAAGGCT-3'; GAPDH-R, 5'-TC AGGTCCACCACTGACACGTT-3' $(17,18)$. PCR was carried out using Takara ExTaq (Takara, Tokyo, Japan) according to the manufacturer's instructions with the following cycle conditions: GAPDH, $94^{\circ} \mathrm{C}$ for $2 \mathrm{~min}(1$ cycle $), 94^{\circ} \mathrm{C}$ for $30 \mathrm{sec}, 55^{\circ} \mathrm{C}$ for $30 \mathrm{sec}, 72^{\circ} \mathrm{C}$ for $40 \mathrm{sec}(30$ cycles $), 72^{\circ} \mathrm{C}$ for $10 \mathrm{~min}$ ( 1 cycle), TLR 2 and TLR $3,94^{\circ} \mathrm{C}$ for $2 \mathrm{~min}$ ( 1 cycle), $94^{\circ} \mathrm{C}$ for $30 \mathrm{sec}, 57^{\circ} \mathrm{C}$ for $30 \mathrm{sec}, 72^{\circ} \mathrm{C}$ for $40 \mathrm{sec}(32 \mathrm{cycles})$, $72^{\circ} \mathrm{C}$ for $10 \mathrm{~min}(1 \mathrm{cycle}), \mathrm{TLR} 4$ and TLR $9,94^{\circ} \mathrm{C}$ for $2 \mathrm{~min}$ (1 cycle), $94^{\circ} \mathrm{C}$ for $30 \mathrm{sec}, 58^{\circ} \mathrm{C}$ for $30 \mathrm{sec}, 72^{\circ} \mathrm{C}$ for $40 \mathrm{sec}$ (30 cycles), $72^{\circ} \mathrm{C}$ for $10 \mathrm{~min}\left(1\right.$ cycle), survivin, $94^{\circ} \mathrm{C}$ for $2 \mathrm{~min}, 94^{\circ} \mathrm{C}$ for $40 \mathrm{sec}, 60^{\circ} \mathrm{C}$ for $1 \mathrm{~min}, 72^{\circ} \mathrm{C} 1 \mathrm{~min}$ ( 30 cycles), $72^{\circ} \mathrm{C}$ for 7 min $(1$ cycle $)(17,18)$. Products were resolved on $1.8 \%$ agarose gels and visualized by ethidium bromide staining.
Quantitative real-time PCR. Quantification of mRNA levels of the target gene was performed using real-time fluorescence detection TaqMan technology and GeneAmp 7700 Sequence Detector (PE Applied Biosystems). The theoretical and practical aspects of real-time quantitative PCR have been described elsewhere. Target cDNA levels were quantified by measuring $\mathrm{Ct}$ (threshold cycle). The parameter $\mathrm{Ct}$ is defined as the fractional cycle number at which the fluorescence generated by cleavage of a dual-labeled probe is detectable by the detector. Optimal primer and probe sequences were determined using Primer Express (PE Applied Biosystems). The primers and TaqMan probe for human $B$-actin (Pre-developed TaqMan Assay Reagents Endogenous control) and TLR3 mRNA (Hs00152933) were purchased from PE Applied Biosystems. The quantification of TLR3 mRNA expression was examined by following the manufacturer's instructions. In this quantitative PCR system, fluorescent emission is measured in real-time and the calculated $\mathrm{Ct}$ reflects the starting target quantity. Relative gene expression was determined according to the $\Delta \Delta \mathrm{Ct}$ methods. The amount of target, normalized to the endogenous reference and relative to a calibrator, was given by diluted HSC3 (1:100).

Western blot analysis. Western blot analysis was performed to determine the expression of TLR2, TLR3 and survivin. After cellular extraction with $1 \mathrm{ml}$ of $0.25 \%$ tripsin/EDTA, cell lysates were mixed in loading buffer with 5\% 2ME and boiled for $5 \mathrm{~min}$. Then, samples were subjected to $14 \%$ SDSPAGE. After electrophoresis, proteins were transferred onto a PVDF membrane (Millipore, Billerica, MA, USA). The blots were incubated with mouse anti-human monoclonal TLR2 and TLR3 antibodies (Santa Cruz Biotechnology, Inc., USA) for $1 \mathrm{~h}$ at room temperature. Membranes were washed with $0.5 \%$ Tween TBS and incubated with HRP-linked antimouse IgG secondary antibody (Santa Cruz Biotechnology, Inc.) for $30 \mathrm{~min}$ at room temperature. To examine the expression of survivin, Western blot analysis was performed with rabbit anti-human survivin monoclonal antibody (Alpha Diagnostic Int. Inc., TX, USA) for $1 \mathrm{~h}$ at room temperature, and goat HRP-linked anti-rabbit IgG secondary antibody (Santa Cruz Biotechnology, Inc.) for $30 \mathrm{~min}$ at room temperature. Protein bands were visualized with ECL substrate (GE Medical).

Flow cytometry. To detect cell surface expression, cultured human tumor cells were stained with purified mouse antihuman TLR2 antibody (Santa Cruz Biotechnology, Inc.) and purified mouse anti-human TLR3 antibody (Santa Cruz Biotechnology, Inc.). Purified mouse IgG2 control (Santa Cruz Biotechnology, Inc.), followed by FITC-conjugated rat anti-mouse IgG2 monoclonal antibody (Santa Cruz Biotechnology, Inc.), and purified mouse IgG1 control followed by FITC-conjugated goat anti-mouse IgG1 monoclonal antibody (Santa Cruz Biotechnology, Inc.) were also used for staining.

To analyze intracytoplasmic TLR3 expression, tumor cells were prefixed and permeabilized using IntraPrep (Beckman Coulter, USA). The staining treatments were conducted as above. Finally, stained cells were analyzed using a flow cytometer (FACS Calibur, Becton-Dickinson, 
A



B

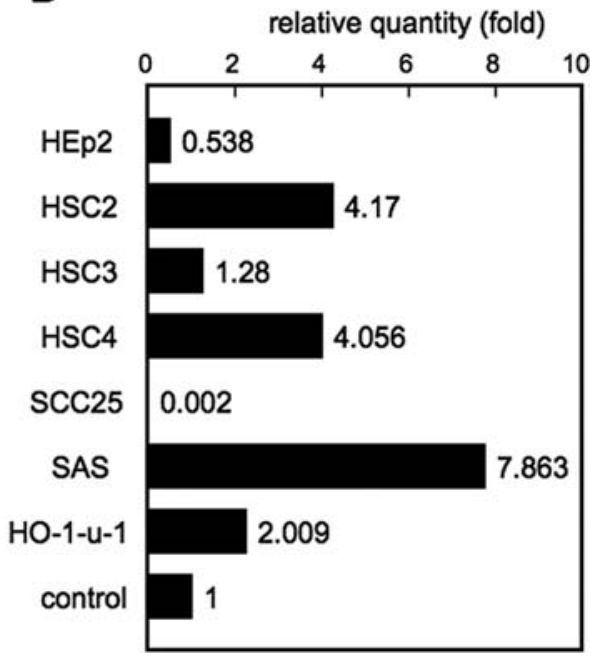

Figure 1. Expression of TLR mRNA in HNSCCs. (A) TLR expression at mRNAs levels were obtained from 8 cell lines. Cells were cultured, mRNA was extracted and then RT-PCR was performed. TLR2 and TLR3 showed varied expression in head and neck cancer at mRNA level. Most cell lines tended to show strong expression of TLR2 and TLR3 mRNA and weak expression of TLR4 and TLR9 mRNA. (B) Quantitative RT-PCR of TLR3 was performed. Diluted HSC-3 (1:100) was used as a control. SAS showed strong TLR3 expression, HO-1-u-1 showed moderate expression, and SCC25 showed weak expression.

Franklin Lakes, NJ, USA), and the data were processed with Cell Quest software.

Cell stimulation experiments and apoptosis assays. Tumor cells were plated prior to stimulation at subconfluent densities. Media were replaced with fresh media and 10 and $100 \mu \mathrm{g} / \mathrm{ml}$ poly I:C (InvivoGen) or control was added. To evaluate apoptosis, MTT assay and flow cytometry were performed. For the cell viability assay, $10^{3}$ or $10^{4}$ cells were plated into 96-well plates and incubated with 10 and $100 \mu \mathrm{g} / \mathrm{ml}$ poly I:C or control for $24 \mathrm{~h}$. MTT cell growth kit (Chemicon International, CA, USA) was used and the assay followed recommended experimental methods. Flow cytometry was conducted by pre-culture of the cells with poly I:C for $24 \mathrm{~h}$, and then $10^{5}$ cells were washed twice with cold PBS, followed by resuspension in binding buffer. Cells were then incubated with FITC-conjugated Annexin V and propidium iodide (PI) in darkness for $20 \mathrm{~min}$ at room temperature. Samples were immediately analyzed by a FACS Calibur flow cytometer. Moreover, to clarify the mechanism of apoptosis, RT-PCR and Western blot analysis were performed. Cells were incubated with 10 and $100 \mu \mathrm{g} / \mathrm{ml}$ poly I:C or control for $24 \mathrm{~h}$, and the expression of survivin was detected.

Statistics. Mann-Whitney U-test was used to determine differences between the data.

\section{Results}

Expression of TLR mRNA in HNSCCs. We tested various human HNSCC cell lines, including HEp2, SCC25, HSC2, HSC3, HSC4, SAS, HSQ89 and HO-1-u-1 for the expression of TLR2, TLR3, TLR4 and TLR9 by RT-PCR. Cell lines showed varied expression of TLRs; however, most cell lines tended to show strong TLR2 and TLR3 mRNA expression and weak TLR4 and TLR9 expression (Fig. 1A). Quantitative
RT-PCR of TLR3 was also performed (Fig. 1B). Diluted HSC-3 (1:100) was used as control. SAS showed strong TLR3 expression, HSC-2, HSC-4 and HO-1-u-1 showed moderate expression, and HEp2 and SCC25 showed weak expression.

Expression of TLR protein in HNSCCs. The expression of TLR 2 and TLR 3 was also examined by Western blot analysis and flow cytometry. TLR2 and TLR3 were also expressed in most cell lines at the protein level (Fig. 2A). Flow cytometry with SAS showed that TLR3 was expressed mainly in the intracellular compartments (Fig. 2B). Similar results showing that TLR2 and TLR3 were expressed in HNSCCs were obtained in these four independent experiments (Figs. 1 and 2). The broad expression of TLRs in tumor cells suggests that TLRs may have a previously unrecognized function in tumor biology.

TLR3 agonist-induced apoptosis in HNSCC cell lines. TLR3 was expressed strongly in SAS, moderately in HO-1-u-1 and weakly in SCC25. To clarify the function of TLRs in tumor cells, we incubated these cells with poly I:C, the ligand for TLR3. Interestingly, after incubation of SAS, HO-1-u-1 and SCC25 with poly I:C for $24 \mathrm{~h}$, these tumor cells showed changes in the number of cells and cell formation. Moreover, the changes were dependent on TLR3 expression (Fig. 3). Cell viability was evaluated by MTT assays. Twenty-four hours after incubation with $100 \mu \mathrm{g} / \mathrm{ml}$ poly I:C, SAS decreased to $38.2 \%$, HO-1-u-1 decreased to $67.0 \%$, and SCC25 showed a slight decrease (Fig. 4). In TLR3-expressing cell lines, the stimulation of TLR3 induced a reduction in cell numbers in a poly I:C dose-dependent manner. Moreover, to evaluate the apoptosis of tumor cells, cell lines were stimulated by poly I:C for $24 \mathrm{~h}$, and flow cytometry with Annexin V and PI staining was performed. The apoptotic cells, Annexin V-positive and PI-negative cells, were observed in $24.1 \%$ cells in SAS and $14.1 \%$ cells in HO-1-u-1; however, few apoptotic changes 
A

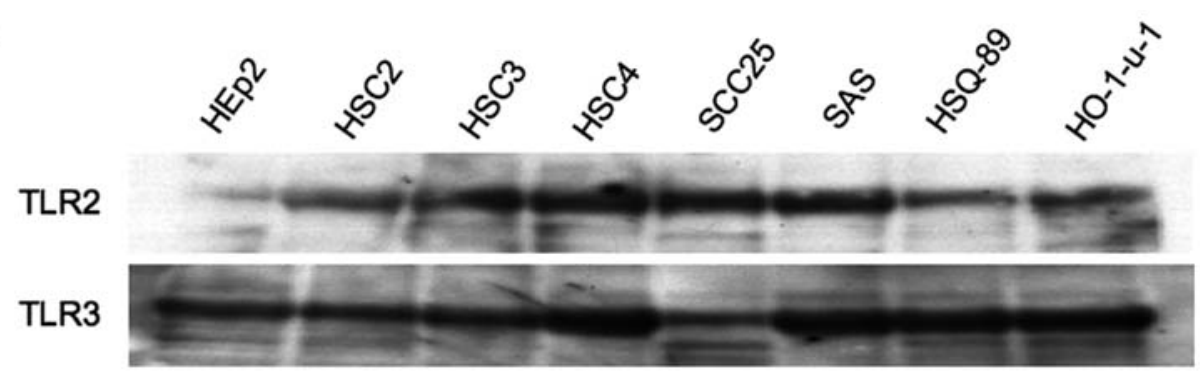

B
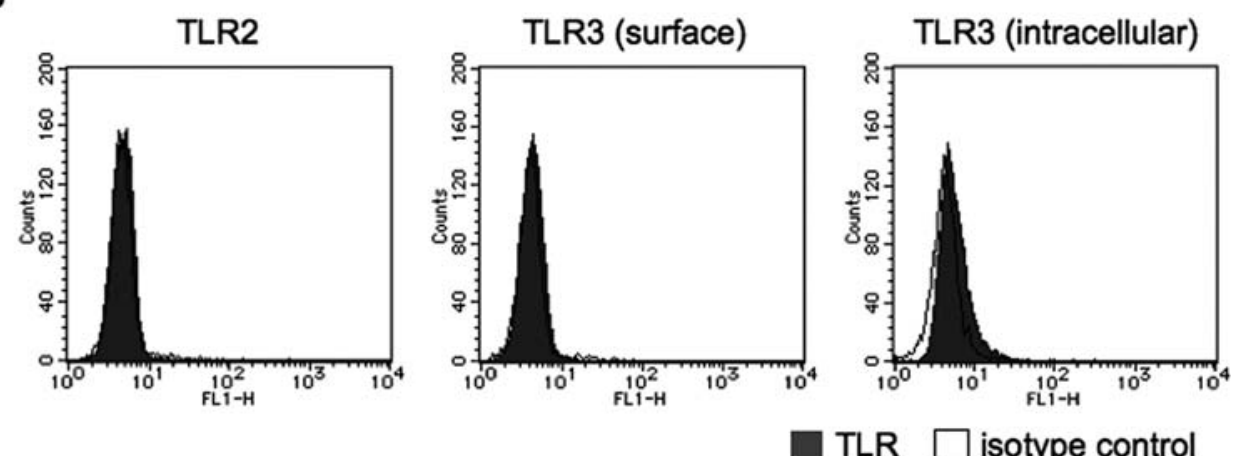

Figure 2. Expression of TLR protein in HNSCCs. (A) Western blot analysis of TLR2 and TLR3 expressions in extracts obtained from the same cell lines were performed. Cells were cultured and whole-cell lysates were subjected to Western blot analysis for TLR2 and TLR3. Varied expression of TLR2 and TLR3 was found in head and neck cancer at the protein level. (B) TLR2 and TLR3 expression was analyzed by flow cytometry. SAS, which strongly expressed TLR3, was cultured and stained with fluorescence antibodies. TLR3 was examined on both the intracellular components and cell surfaces. TLR3 is mainly expressed on the intracellular components in head and neck cancer.

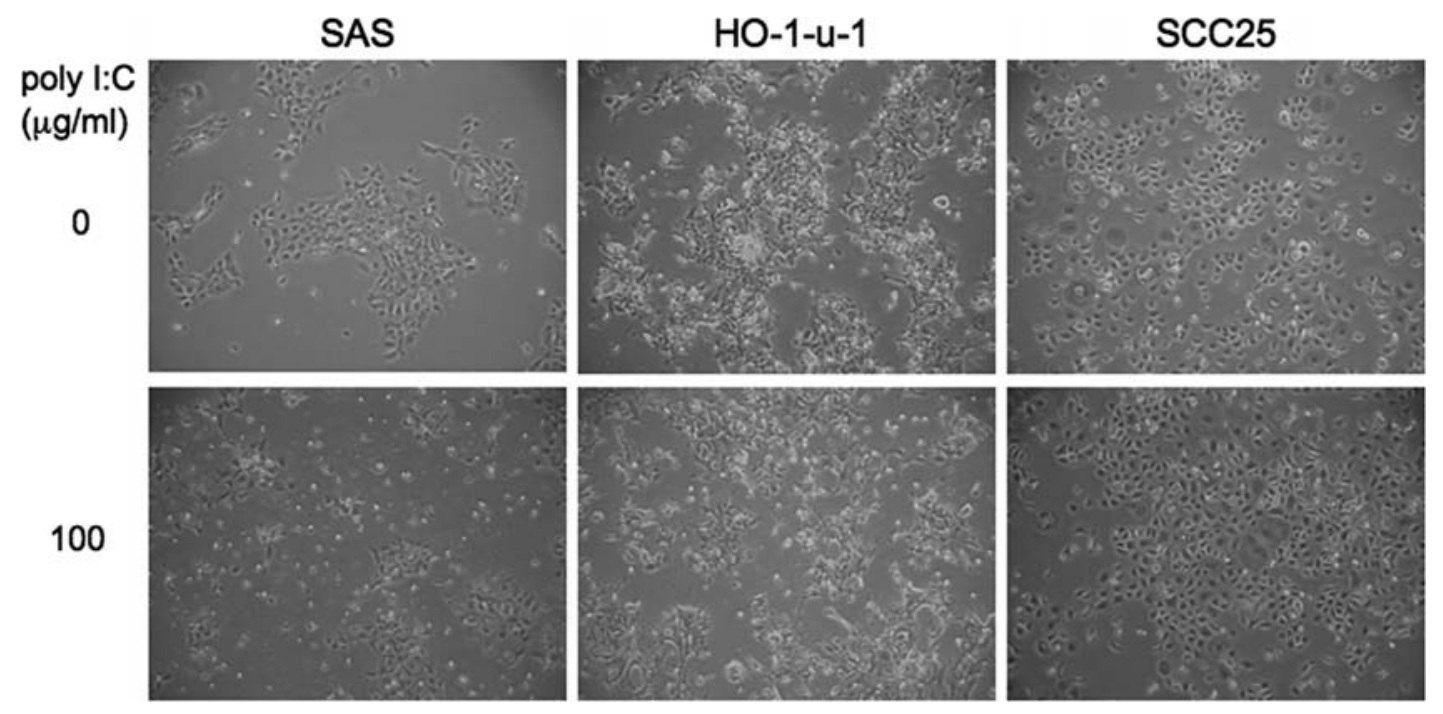

Figure 3. Cell changes with poly I:C stimulation. SAS, HO-1-u-1 and SCC25 were cultured to subconfluent states and then stimulated with $100 \mu 1 / \mathrm{ml}$ poly I:C for $24 \mathrm{~h}$. SAS cell numbers decreased and the cell shapes were also changed to shrunken forms. HO-1-u-1 showed moderate changes. However, few changes occurred in SCC25.

occurred in SCC (2.2\%). These results suggest that poly I:C directly induced apoptosis through a TLR3 pathway.

Poly I:C induces cell apoptosis via survivin pathways. To elucidate the mechanism for TLR3-mediated tumor cell apoptosis, the expression of survivin was analyzed by RT-PCR and Western blot assays. HNSCC cell lines showed strong survivin expression without poly I:C stimulation; however, the expression of survivin was down-regulated in this apoptotic process with poly I:C stimulation, both at the mRNA and protein levels. Down-regulation of survivin occurred strongly in SAS, followed by HO-1-u-1, but the expression of survivin was not down-regulated with poly I:C stimulation in SCC25 (Fig. 6). These changes corresponded to TLR3 expression, in a poly I:C dose-dependent manner. The results indicate that poly I:C induced apoptosis through TLR3 pathways, and 


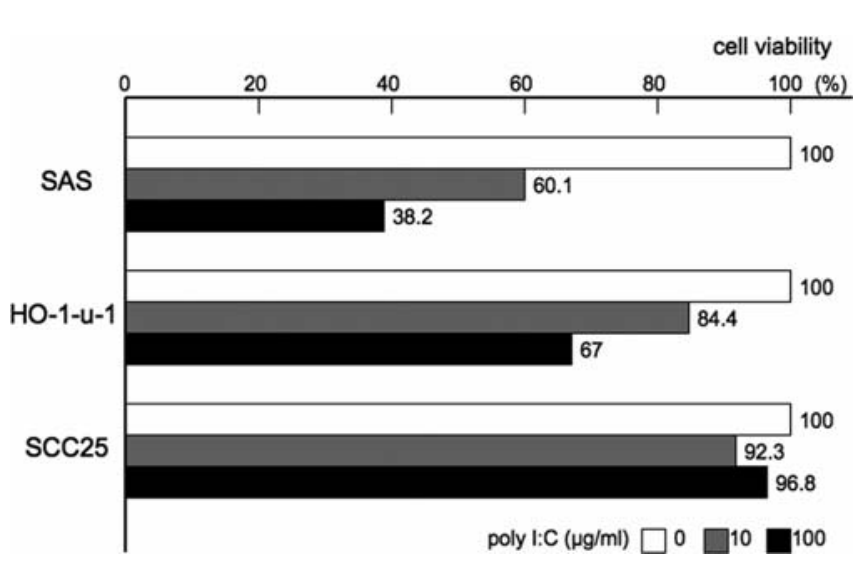

Figure 4. MTT assay. To evaluate the decrease in numbers of cells, MTT assay was also performed. SAS, HO-1-u- 1 and SCC25 cells $\left(10^{3}\right.$ or $\left.10^{4}\right)$ were plated onto 96 -well plates and stimulated with 10 or $100 \mu \mathrm{l} / \mathrm{ml}$ poly $\mathrm{I}$ :C for $24 \mathrm{~h}$. As a control group, cells were cultured without poly I:C stimulation. MTT assay was performed by following recommended experimental methods. In SAS, and HO-1-u-1, which expressed TLR3, cell numbers decreased in a poly I:C dose-dependent manner. In contrast, few changes occurred in SCC25.

depended on the survivin mediated pathway in HNSCC cell lines.

\section{Discussion}

In the present study, we demonstrated that many HNSCC cells expressed TLR at the cellular and transcriptional levels and that TLR3-stimulation induced apoptosis in TLR3expressing cancer cells. The mammalian immune system is composed of two branches: innate and acquired immunity. The innate immune response is the first line of host defense against pathogens and discriminates between self and a variety of pathogens. On the other hand, acquired immunity is characterized by specificity and is involved in the elimination of pathogens in the late phase of infection, as well as the generation of immunological memory. TLRs are important sensors in the innate immune system and are thought to be mainly expressed in immune cells. The ligation of TLRs by their ligands represents an important link between innate and adaptive immune responses. Therefore, TLRs are also thought to be important triggers of immunological responses. TLR3 is the critical sensor of the innate immune system, and serves to identify viral double-stranded RNA (dsRNA). dsRNA is produced by many viruses during replication (19). TLR3 is reported to be expressed on immune cells, specifically in conventional dendritic cells. Furthermore, TLR3 is also expressed in a variety of epithelial cells, including airway, uterine, corneal, vaginal, cervical, biliary and intestinal epithelial cells, which all function as efficient barriers to infection (20). Recent studies reported that certain human tumor cells also express TLRs. It is an interesting paradox that TLRs are expressed in invasive cancerous cells. TLR3 is expressed in breast cancer (10), melanoma (11), prostate cancer (12), cervical cancer (13), colon cancer (13), hepatocellular carcinoma (14) and head and neck cancer (15), while TLR4 is expressed in lung cancer (17). In HNSCCs, studies of TLR expression have been reported by only two investigators $(15,16)$. Pries and his colleagues reported TLR3 expression and signaling in HNSCCs (15). Moreover, He and his colleagues reported evidence of an association between a TLR3 sequence variant and a risk of carcinogenesis in

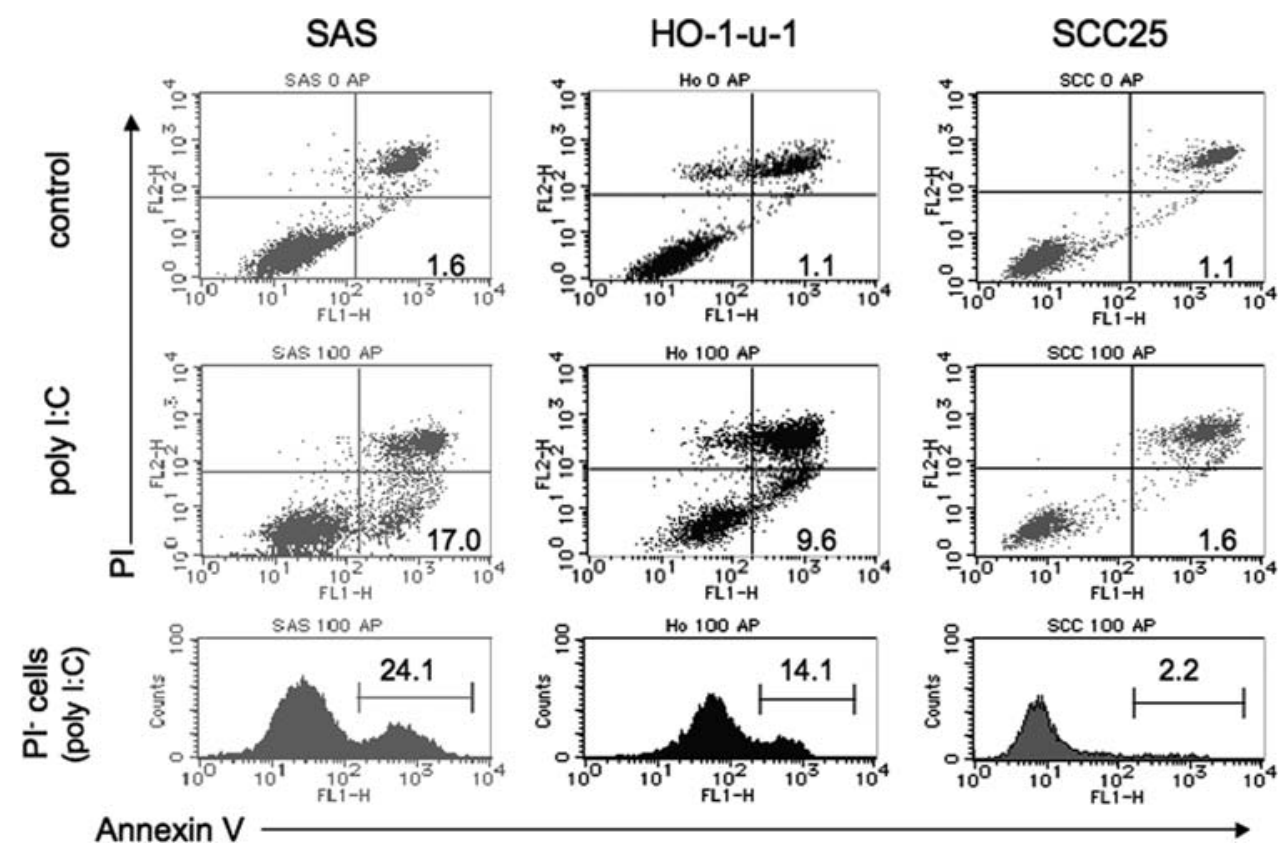

Figure 5. Flow cytometry with Annexin V and PI staining. Flow cytometry was performed to evaluate apoptosis. Cells were pre-cultured with poly I:C for $24 \mathrm{~h}$, and $10^{5}$ cells were washed twice with cold PBS, followed by resuspension in binding buffer, and then incubation with FITC-conjugated Annexin-V and propidium iodide (PI). Samples were immediately analyzed using a FACS Calibur flow cytometer. Apoptotic cells, Annexin V-positive and PI-negative cells were detected in TLR3-expressing cells. Similar results were obtained in three independent experiments (Figs. 3-5). Stimulation of TLR3 directly induced cell apoptosis in a poly I:C-dependent manner. 


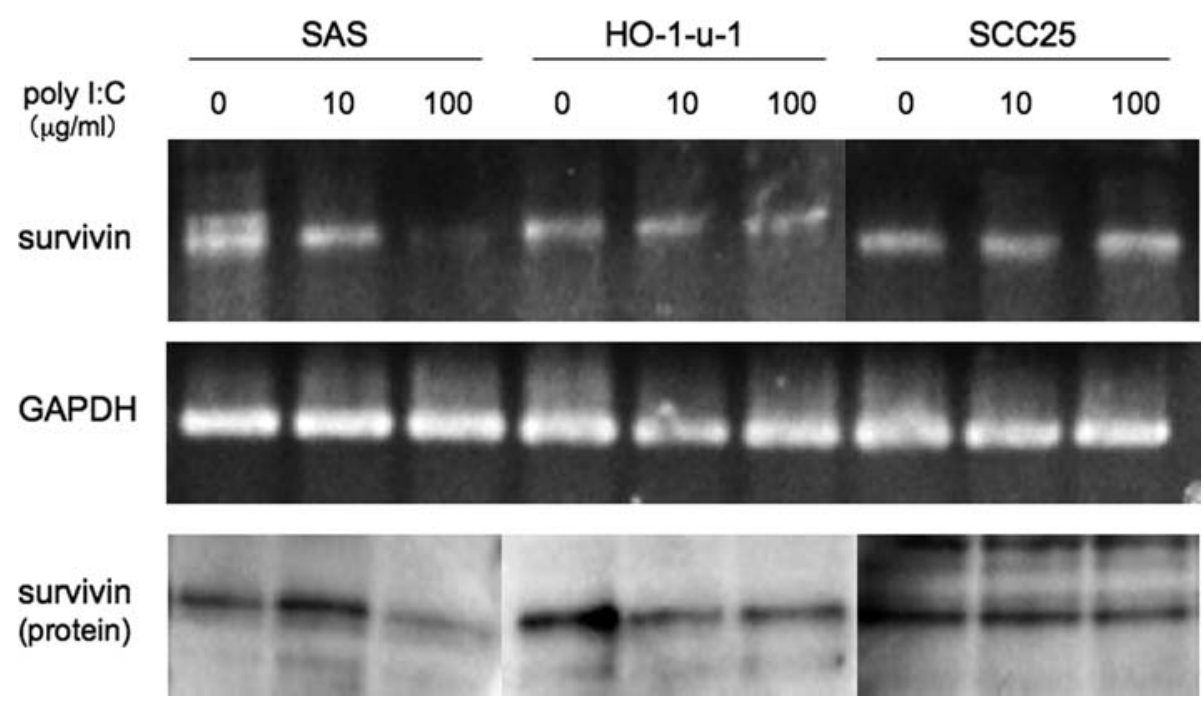

Figure 6. Poly I:C induces cell apoptosis via survivin pathways. The expression of survivin was examined to elucidate the mechanism of apoptosis induced by TLR3 stimulation. Twenty-four hours after incubation with 0,10 and $100 \mu \mathrm{g} / \mathrm{ml}$ poly I:C, RT-PCR and Western blot analysis for determining survivin expression were performed. Survivin was strongly expressed in control groups; however, the expression of survivin was down-regulated during apoptosis both at the mRNA and protein levels.

nasopharyngeal carcinoma (16). In this study, we clarified that HNSCCs showed varied TLR expression and that they tended to show strong TLR3 expression. Our study suggests that TLR3 might play a role in the tumor biology of HNSCCs.

The ligation of TLR3 and its ligand dsRNA triggers wellcharacterized signaling cascades that result in activation of downstream effectors, such as NF- $\mathrm{KB}$, p38, JNK and IFN regulatory factors (IRFs) 4 (1). The innate immune system is thought to be mediated by phagocytes, including macrophages and dendritic cells, and these cells and various inflammatory cytokines are involved in these signaling cascades. These unique pathways also induce T-cell activation and differentiation, which result in immune responses against tumor cells. On the other hand, an investigator has recently reported that stimulation of TLR 3 could directly cause tumor cell apoptosis (10-14). In breast cancer (10), melanoma (11), prostate cancer (12), cervical cancer (13), colon cancer (13) and hepatocellular carcinoma (14), TLR3 directly induced cell apoptosis with poly I:C. However, only one study was reported in HNSCCs. Pries et al reported that HNSCC cell lines expressed TLR3 and that the expression of onco-protein c-Myc was significantly decreased in TLR3 siRNA-transfected HNSCC cells, suggesting that TLR3 might participate in tumor cell proliferation (15). In the present study, we investigated the response of HNSCC cell lines in vitro to stimulation with TLR3 agonist poly I:C. Interestingly, in TLR3-expressing cell lines, the ligation of TLR3 by poly I:C directly caused apoptosis of the cells in a poly $\mathrm{I}: \mathrm{C}$ dose-dependent manner. This result was in conflict with previous data in HNSCCs. It might be because different cell lines were included in our study; however, our results were similar to most other data. In non-cancerous cells, poly I:C, represents either genomic or life cycle intermediate material of many viruses, and activates the immune cells through binding to both dsRNA-dependent protein kinase (PKR) and TLR3. On the other hand, double-stranded RNA has also been proven to induce apoptosis in several cell types through multiple pathways. Scarim et al reported that
dsRNA-transfected pancreatic $\beta$-cells manifest PKR- and caspase-dependent apoptosis $(21,22)$, whereas endothelial cell apoptosis triggered by exogenous dsRNA is mostly dependent on the extrinsic caspase pathway $(19,20)$. As the involvement of TRIF in apoptosis has recently been suggested $(23,24)$, the TLR3 signaling pathway not only participates in limiting virus replication, but also causes infected cells to undergo apoptosis, which is another way of protecting the host against the spread of microbes (25) in TLR3-expressing non-cancerous cells. The current study demonstrated that TLR3-stimulation directly induced apoptosis in TLR3expressing cancer cells. Our results suggest a new clinical perspective for TLR3-mediated multifunctional signaling, which can both directly kill the cancer cells and induce innate and specific immune antitumor responses.

Another important point of the current study is the direct demonstration of the involvement of survivin in TLR3related apoptosis. This is the first report to show that survivin is involved in TLR3-related tumor cell apoptosis. Survivin is a member of the inhibitor of apoptosis protein (IAP) family which has effects on both the extrinsic and intrinsic caspase pathways by binding to some caspases (26). The survivin protein is abundantly expressed during fetal development in humans, but rarely present in adult tissues. However, the expression of survivin has been reported in most human tumors, suggesting that alterations in survivin gene regulation commonly occurs during tumorigenesis $(18,26)$. Survivin is expressed in G2-M in a cell cycle-dependent manner, and binds directly to mitotic spindle microtubules, suggesting a role of survivin as a novel apoptotic checkpoint during cell division (27). Therefore, survivin is thought to be a new biomarker or therapeutic target for cancer patients. Survivin is an important inhibitor of apoptosis, especially as a direct inhibitor of caspases-3 and 7 (28). There are 11 caspases in humans, and many, such as caspases-2, 3, 6, 7, 8, 9, 10 and 12 , are implicated in apoptosis; however, some, such as caspases-1, 2, 3 and 4 also exert non-apoptotic functions, 
including cell proliferation, differentiation and NF- $\mathrm{KB}$ activation. Maelfait et al reported the roles of caspase-1 and caspase- 8 in the production of biologically active IL- 13 in response to TLR3 and TLR4 stimulation in the immune response of human embryonic kidney (29). On the other hand, Salaun et al reported that TLR3 triggered apoptosis in breast cancer cell lines directly via the TRIF-dependent pathway (10). The immunological characteristics of noncancerous and cancer cells might show differences; however, these TRIF- and caspase-dependent pathways might evolve in tumor apoptosis with TLR3 stimulation. Apoptosis of TLR3-expressing HNSCC cells might be mostly dependent on the TRIF-dependent extrinsic caspase pathway.

Overall, the ligation of TLR3 by poly I:C directly caused apoptosis of these cells and the expression of survivin was down-regulated during apoptosis. These results suggest that TLR3 might be a new therapeutic target for HNSCC patients. A combination of these therapies and surgery or radiotherapy might be useful treatments for head and neck cancers.

In conclusion, we reported that the stimulation of TLR3 directly caused apoptosis of HNSCC cells, and that the expression of survivin was down-regulated during this process. TLR 3 and its signaling might be significant for clarification of the tumor biology in HNSCCs. Furthermore, TLR3 might also be a new strategy for the treatment of HNSCC patients.

\section{Acknowledgements}

We thank Dr N. Uemura for the technical supports for cell culture, Mrs. Y. Takenaka, Mrs. C. Shibatomi for the preparation of regents, and Mrs. A. Miura and Ms. Y. Hirai for preparation of the manuscript.

\section{References}

1. Akira S and Takeda K: Toll-like receptor signaling. Nat Rev Immunol 4: 499-511, 2004

2. Takeda K and Akira S: TLR signaling pathways. Semin Immunol 16: 3-9, 2004

3. Kawai $\mathrm{T}$ and Akira S: Innate immune recognition of viral infection. Nat Immunol 7: 131-137, 2006.

4. Diebold SS, Kaisho T, Hemmi H, Akira S, Reis E and Sousa C: Innate antiviral responses by means of TLR7-mediated recognition of single-stranded RNA. Science 303: 1529-1531, 2004.

5. Heil F, Hemmi H, Hochrein H, et al: Species-specific recognition of single-stranded RNA via toll-like receptor 7 and 8 . Science 303: 1526-1529, 2004

6. Hornung V, Guenthner-Biller M, Bourquin C, et al: Sequencespecific potent induction of IFN-alpha by short interfering RNA in plasmacytoid dendritic cells through TLR7. Nat Med 11: 263-270, 2005

7. Ahmad-Nejad P, Hacker H, Rutz M, Bauer S, Vabulas RM and Wagner H: Bacterial CpG-DNA and lipopolysaccharides activate toll-like receptors at distinct cellular compartments. Eur J Immunol 32: 1958-1968, 2002.

8. Alexopoulou L, Holt AC, Medzhitov R and Flavell RA: Recognition of double-stranded RNA and activation of NF-kappaB by toll-like receptor 3. Nature 413: 732-738, 2001.

9. Kariko K, Bhuyan P, Capodici J and Weissman D: Small interfering RNAs mediate sequence-independent gene suppression and induce immune activation by signaling through toll-like receptor 3. J Immunol 172: 6545-6549, 2004.
10. Salaun B, Coste I, Rissoan MC, Lebecque SJ and Renno T: TLR3 can directly trigger apoptosis in human cancer cells. J Immunol 176: 4894-4901, 2006.

11. Alaun B, Lebecque S, Matikainen S, Rimoldi D and Romero P: Toll-like receptor 3 expressed by melanoma cells as a target for therapy? Clin Cancer Res 13: 4565-4574, 2007.

12. Paone A, Starace D, Galli R, et al: Toll-like receptor 3 triggers apoptosis of human prostate cancer cells through a PKC-alphadependent mechanism. Carcinogenesis 29: 1334-1342, 2008.

13. Jiang Q, Wei H and Tian Z: Poly I:C enhances cycloheximideinduced apoptosis of tumor cells through TLR3 pathway. BMC Cancer 8: 12, 2008.

14. Khavalevsky E, Rivkin L, Rashmilewitz J, Galun E and Giladi H: TLR3 Signaling in a hepatoma cell line is skewed towards apoptosis. J Cell Biochem 100: 1301-1312, 2007.

15. Pries R, Hogrefe L, Xie L, Frenzel H, Brocks C, Ditz C and Wollenber B: Induction of c-Myc-dependent cell proliferation through toll-like receptor 3 in head and neck cancer. Int J Mol Med 21: 209-215, 2008.

16. He JF, Jia WH, Fan Q, Zhou XX, Qin HD, Shugart YY and Zeng YX: Genetic polymorphisms of TLR3 are associated with nasophryngeal carcinoma risk in Cantonese population. BMC Cancer 7: 194, 2007.

17. He W, Li Qu, Wang L, Chen W, Li N and Cao X: TLR4 signaling promotes immune escape of human lung cancer cells by inducing immunosuppressive cytokines and apoptosis resistance. Mol Immunol 44: 2850-2859, 2007.

18. Satoh K, Kaneko K, Hirota M, Masamune A, Satoh A and Shimosegawa T: Expression of survivin is correlated with cancer cell apoptosis and is involved in the development of human pancreatic duct cell tumors. Cancer 92: 271-278, 2007.

19. Kaiser WJ, Kaufman JL and Offermann MK: IFN-alpha sensitizes human umbilical vein endothelial cells to apoptosis induced by double-stranded RNA. J Immunol 172: 1699-1710, 2004.

20. Sato A, Iizuka M, Nakagomi O, et al: Rotavirus double-stranded RNA induces apoptosis and diminishes wound repair in rat intestinal epithelial cells. J Gastroenterol Hepatol 21: 521-530, 2006.

21. Scarim AL, Arnush M, Blair LA, et al: Mechanisms of beta-cell death in response to double-stranded (ds) RNA and interferongamma: dsRNA-dependent protein kinase apoptosis and nitric oxide-dependent necrosis. Am J Pathol 159: 273-283, 2001.

22. Robbins MA, Maksumova L, Pocock E and Chantler JK: Nuclear factor-kappaB translocation mediates double-stranded ribonucleic acid-induced NIT-1 beta-cell apoptosis and upregulates caspase-12 and tumor necrosis factor receptorassociated ligand (TRAIL). Endocrinol 144: 4616-4625, 2003.

23. Ruckdeschel K, Pfaffinger G, Haase R, et al: Signaling of apoptosis through TLRs critically involves toll/IL-1 receptor domain-containing adapter inducing IFN-beta, but not MyD88, in bacteria-infected murine macrophages. J Immunol 173: 3320-3328, 2004.

24. Kaiser WJ and Offermann MK: Apoptosis induced by the tolllike receptor adaptor TRIF is dependent on its receptor interacting protein homotypic interaction motif. J Immunol 174: 4942-4952, 2005.

25. Everett $\mathrm{H}$ and McFadden G: Apoptosis: an innate immune response to virus infection. Trends Microbiol 7: 160-165, 1999.

26. Ambrosini G, Adida C and Altireri DC: A novel anti-apoptosis gene, survivin, expressed in cancer and lymphoma. Nat Med 3: 917-921, 1997.

27. Li F, Ambrosini G, Chu EY, Plescia J, Tognin S, Marchisio PC and Altieri DC: Control of apoptosis and mitotic spindle checkpoint by survivin. Nature 396: 580-584, 1998.

28. Deveraux QL, Takahashi R, Salvesen GS and Reed JC: X-linked IAP is a direct inhibitor of cell-death proteases. Nature 388: 300-304, 1997.

29. Maelfait J, Vercammen E, Janssens S, Schotte P, Haegman M, Magez S and Beyaert R: Stimulation of toll-like receptor 3 and 4 induces interleukin-1beta maturation by caspase- 8 . J Exp Med 205: 1967-1973, 2008. 